\title{
Sociodemographic differences in reasons for ENDS use among US youth within Wave 2 of the PATH study
}

\author{
Connie Xiao ${ }^{1}$, Kathryn Heley², Ryan David Kennedy ${ }^{3,4}$, Lisa Lagasse ${ }^{3,4}$, Meghan Bridgid Moran ${ }^{4}$
}

\begin{abstract}
INTRODUCTION Adolescents use electronic nicotine delivery systems (ENDS, or e-cigarettes) more than other tobacco products. Among adults, some data indicate that motivations for use vary by sociodemographic group. This study sought to examine how adolescents' motivations for ENDS use vary by sociodemographic characteristics, including age, gender, race/ethnicity and household income.

METHOds The current study used data from Wave 2 of the Population Assessment of Tobacco and Health (PATH) study. Youth who used ENDS in the past 30 days were asked to report their motivations for product use. Rates of reporting each reason for use were compared across sociodemographic groups.

RESULTS Appealing flavors was the most commonly reported motivation for using ENDS, and was mentioned more often among females $(89.23 \%)$ than males $(74.00 \%)$. Females were also more likely than males to report using ENDS because the product feels like smoking cigarettes $(\mathrm{AOR}=1.761)$ and people who are important to the participant smoke them $(\mathrm{AOR}=1.895)$. Older teens were more likely to report using ENDS because the product does not smell bad (56.45\%, 15-17 years old; $42.83 \%, 12-14$ years old).

CONCLUSIONS Motivations for ENDS use vary by sociodemographic group. Understanding the motivations for use among sociodemographic subgroups is an initial step towards informing the development of policies and interventions with equally distributed benefits.
\end{abstract}

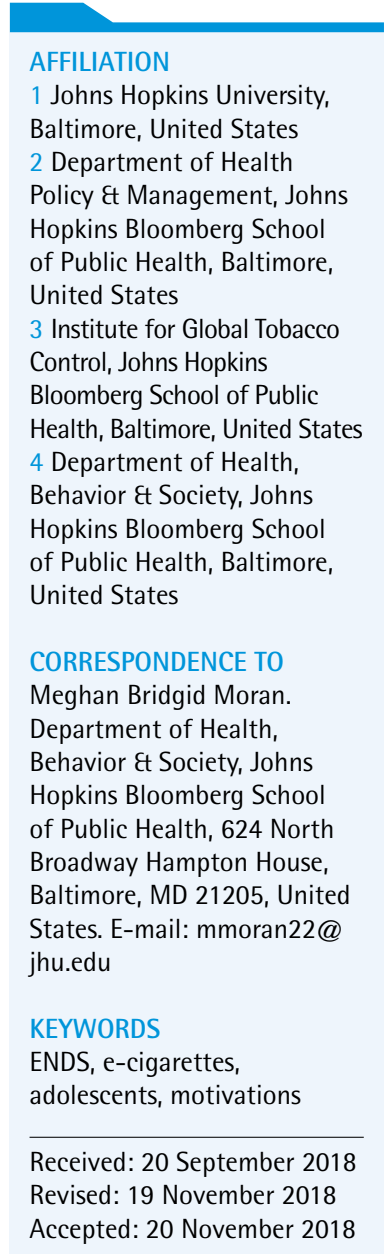

\section{INTRODUCTION}

Recent data indicate that American youths' use of electronic nicotine delivery systems (ENDS) exceeds that of other tobacco products ${ }^{1}$. As of $2017,11.7 \%$ of high schoolers and $3.3 \%$ of middle schoolers used ENDS in the past 30 days $^{1}$. Research examining sociodemographic differences in ENDS use indicates that males ${ }^{2}$, adolescents of higher socioeconomic status ${ }^{3}$, and non-Hispanic White youth ${ }^{4}$ may be more likely to use ENDS. Although ENDS have been positioned as potential harm reduction tools for combustible cigarette smokers ${ }^{5}$, their use among adolescents remains problematic as ENDS solutions and aerosols contain numerous toxicants and carcinogens ${ }^{6}$, in addition to nicotine, an addictive substance with neurotoxic effects on the developing brain? 
Studies have identified several reasons for ENDS' increasing popularity among adolescents, including greater visibility in the media ${ }^{8}$, combustible cigarette smoking cessation ${ }^{9}$, interest in flavors ${ }^{10}$, and peer influence ${ }^{11}$. Like other tobacco products $^{12}$, motivations for ENDS use may vary with sociodemographic characteristics. Studies of US adults demonstrate that appealing flavors are more likely to be a reason for use among young adults ${ }^{13}$, and adult women are more likely to report using e-cigarettes because of their use by friends or family members ${ }^{14}$.

Limited research has examined differences in motivations for ENDS use among sociodemographic subgroups of adolescents. A clear understanding of these variations is valuable to help ensure that messages, interventions and policies are relevant to populations most vulnerable to ENDS use and are culturally tailored as needed. This study examines how adolescents' motivations for ENDS use vary by sociodemographic characteristics, including age, gender, race/ethnicity and household income.

\section{METHODS}

\section{Data}

We analyzed data from the Wave 2 youth sample of the Population Assessment of Tobacco and Health (PATH) study $(\mathrm{N}=12172)^{15}$. PATH was a longitudinal cohort study of US youth and adults and its Wave 2 data collection occurred between 2014-2016. Further study design and sample details are described in Hyland et al. ${ }^{15}$ and the study questionnaire can be found at https://www.icpsr.umich.edu/icpsrweb/NAHDAP/ studies/36231. Our sample for analysis consisted of 415 youth participants who were asked questions about their motivations for ENDS use. These questions were only asked to participants who indicated they had used ENDS in the past 30 days. PATH study procedures were approved by the Westat Institutional Review Board ${ }^{15}$.

\section{Measures}

The primary measures of interest were questions asking youth to identify, from a list of 13 possibilities, the reasons they use ENDS (response options of yes/ no). Table 1 presents the full wording for each item. We examined how motivations varied across several

Table 1. Sample characteristics, United States, 2014-2016 (N=415)

\begin{tabular}{|c|c|c|c|}
\hline & Per cent & $95^{\circ} \mathrm{CI}$ & N (unweighted) \\
\hline \multicolumn{4}{|l|}{ Age (years) } \\
\hline $12-14$ & 19.59 & $(15.65-24.24)$ & 88 \\
\hline $15-17$ & 80.41 & (75.76-84.35) & 327 \\
\hline \multicolumn{4}{|l|}{ Gender } \\
\hline Male & 56.96 & $(51.53-62.24)$ & 237 \\
\hline Female & 43.04 & $(37.76-48.47)$ & 177 \\
\hline \multicolumn{4}{|l|}{ Race } \\
\hline Non-Hispanic White & 69.34 & $(64.24-74.01)$ & 264 \\
\hline Non-Hispanic Black & 5.55 & (3.50-8.68) & 19 \\
\hline Non-Hispanic Other & 8.28 & $(5.84-11.61)$ & 42 \\
\hline Hispanic & 16.83 & $(13.67-20.56)$ & 90 \\
\hline \multicolumn{4}{|l|}{ Household income } \\
\hline Less than $\$ 10000$ & 8.11 & $(5.57-11.65)$ & 31 \\
\hline$\$ 10000-\$ 24999$ & 16.82 & $(13.18-21.22)$ & 75 \\
\hline$\$ 25000-\$ 49999$ & 20.35 & $(16.17-25.29)$ & 76 \\
\hline$\$ 50000-\$ 99999$ & 29.96 & $(25.32-35.05)$ & 110 \\
\hline$\$ 100000$ or more & 24.77 & $(19.56-30.83)$ & 84 \\
\hline \multicolumn{4}{|c|}{ Used flavored e-cigarette in past 30 days } \\
\hline Yes & 79.36 & (74.65-83.39) & 329 \\
\hline No & 10.92 & $(8.2-14.39)$ & 42 \\
\hline Don't know & 9.72 & $(7.18-13.03)$ & 43 \\
\hline
\end{tabular}


Table 1. Continued

\begin{tabular}{|c|c|c|c|}
\hline & Per cent & $95 \% \mathrm{CI}$ & V (unweighted) \\
\hline \multicolumn{4}{|l|}{ Current combustible tobacco user } \\
\hline Non-combustible at W1 and W2 & 46.12 & $(40.95-51.38)$ & 181 \\
\hline Combustible at W1 only & 5.5 & $(3.63-8.27)$ & 22 \\
\hline New W2 combustible (combustible at W2 only) & 23.83 & $(18.95-29.52)$ & 95 \\
\hline Current combustible use at $W 1$ and $W 2$ & 24.54 & $(20.11-29.58)$ & 103 \\
\hline Days used e-cigarette in past 30 days $^{\mathrm{a}}$ & 7.751 & $(6.831-8.672)$ & \\
\hline \multicolumn{4}{|l|}{ Reasons for e-cigarette use } \\
\hline 1. It comes in flavors I like & 77.90 & $(73.07-82.07)$ & 327 \\
\hline 2. They might be less harmful to me than cigarettes & 75.01 & $(70.09-79.37)$ & 306 \\
\hline 3. They might be less harmful to people around me & 74.08 & $(68.61-78.90)$ & 307 \\
\hline 4. It helps people to quit smoking cigarettes ${ }^{b}$ & 63.48 & $(57.22-69.32)$ & 162 \\
\hline 5. They can be used in places where smoking can't & 58.68 & $(53.24-63.92)$ & 242 \\
\hline 6. They don't bother non-tobacco users & 56.40 & $(50.74-61.91)$ & 229 \\
\hline 7. They don't smell & 53.80 & $(48.58-58.93)$ & 229 \\
\hline 8. They are affordable & 48.18 & $(43.91-52.48)$ & 208 \\
\hline 9. I like socializing while using them & 45.80 & $(41.64-50.01)$ & 193 \\
\hline 10. People in the media or other public figures use them & 36.11 & $(30.91-41.65)$ & 150 \\
\hline 11. People who are important to me use them & 34.88 & $(29.69-40.46)$ & 143 \\
\hline 12. It feels like smoking a regular cigarette & 25.09 & $(20.31-30.57)$ & 107 \\
\hline 13. The advertising appeals to me & 14.18 & $(10.63-18.66)$ & 60 \\
\hline
\end{tabular}

a Sample restricted to youth who are past 30-day e-cigarette users. b Asked to youth who last smoked a cigarette within the past year.

sociodemographic characteristics available in the PATH Public Use File: age (12-14, 15-17 years), gender (female, male), race/ethnicity (Hispanic, nonHispanic Black, non-Hispanic White, and other nonHispanic ethnicity), and household income (obtained from the youth's parent: $<\$ 10000, \$ 10000-\$ 24999$, \$25000-\$49999, \$50000-\$99999, $\geq \$ 100000)$.

\section{Data analysis}

We used the weighting and variance estimation procedures provided in the PATH user guide. We calculated descriptive statistics to characterize our sample, then computed crosstabs, with chi-squared tests of significance, to examine bivariate differences in the prevalence of reported motivations for use across identified sociodemographic characteristics. We conducted logistic regression analyses to examine unique associations between sociodemographics and motivations for ENDS use, entering all sociodemographic variables together in models for each motivation, controlling for current combustible tobacco use, intensity of ENDS use (number of days used in past
30 days) and whether the participant uses a flavored ENDS. Analyses were conducted using Stata 14.

\section{RESULTS}

Table 1 presents full descriptive statistics for the analytic sample. The most commonly selected reasons for ENDS use were: appealing flavors (77.9\%), 'they might be less harmful to me than cigarettes' $(75.0 \%)$ and 'they might be less harmful to people around me' (74.1\%). Bivariate analyses revealed several differences across sociodemographic characteristics in motivations for ENDS use (Table 2). Among females, $82.9 \%$ reported using ENDS because the product comes in appealing flavors (compared to $74.0 \%$ of males), 31.3\% reported using ENDS because the product feels like smoking cigarettes (compared to $20.5 \%$ of males), and $43.3 \%$ reported using ENDS because people who are important to them use them (compared to $28.2 \%$ of males). Just over half (53.6\%) of non-Hispanic Black youth reported use due to appealing flavors, compared to non-Hispanic Whites (78.8\%), Hispanics (76.6\%), and those of 
Table 2. Bivariate differences in e-cigarette reasons for use by sociodemographic factors, United States, 2014 $2016(\mathrm{~N}=415)$

\begin{tabular}{|c|c|c|c|c|c|c|c|c|c|}
\hline & \multicolumn{3}{|c|}{ It comes in flavors I like } & \multicolumn{3}{|c|}{$\begin{array}{l}\text { They might be less harmftul to } \\
\text { me than cigaretices }\end{array}$} & \multicolumn{3}{|c|}{$\begin{array}{l}\text { They might be less harmftul to } \\
\text { people around me }\end{array}$} \\
\hline & $\mathrm{N}$ & $\%$ & $95^{\circ} \% \mathrm{CI}$ & $\mathbf{N}$ & $\%$ & $95^{\circ} \mathrm{OCI}$ & N & $\%$ & $95^{\circ} \% \mathrm{CI}$ \\
\hline Overall & 327 & 77.85 & (73.01-82.03) & 306 & 74.96 & (70.02-79.33) & 307 & 748.00 & $(68.61-78.90)$ \\
\hline \multicolumn{10}{|l|}{ Age (years) } \\
\hline $12-14$ & 71 & 79.99 & (70.71-86.88) & 69 & 80.02 & (69.78-87.42) & 68 & 79.32 & (68.17-87.29) \\
\hline 15-17 & 256 & 77.38 & $(71.70-82.21)$ & 237 & 73.81 & (68.04-78.86) & 239 & 72.83 & $(67.21-77.80)$ \\
\hline \multicolumn{10}{|l|}{ Gender } \\
\hline Male & 179 & 74.00 & $(67.79-79.38)^{*}$ & 175 & 75.54 & (68.57-81.39) & 174 & 72.51 & (64.65-79.18) \\
\hline Female & 147 & 82.93 & $(76.02-88.15)^{*}$ & 130 & 74.20 & (67.71-79.78) & 132 & 76.04 & $(68.97-81.93)$ \\
\hline \multicolumn{10}{|l|}{ Race/Ethnicity } \\
\hline Non-Hispanic White & 210 & 78.79 & $(72.88-83.70)^{*}$ & 196 & 74.52 & $(68.31-79.87)$ & 193 & 72.02 & $(65.40-77.81)$ \\
\hline Non-Hispanic Black & 11 & 53.62 & $(27.28-78.09)^{*}$ & 16 & 89.57 & $(67.44-97.27)$ & 14 & 81.03 & $(59.06-92.67)$ \\
\hline Other race & 37 & 89.30 & $(75.69-95.72)^{*}$ & 32 & 78.90 & $(62.72-89.27)$ & 33 & 82.36 & $(65.44-92.01)$ \\
\hline Hispanic & 69 & 76.59 & $(65.42-84.98)^{*}$ & 62 & 70.20 & (58.85-79.52) & 67 & 76.32 & (65.61-84.49) \\
\hline \multicolumn{10}{|l|}{ Household Income } \\
\hline$<\$ 10000$ & 22 & 71.82 & $(53.87-84.77)$ & 22 & 68.38 & (49.20-82.85) & 22 & 71.35 & $(49.16-86.51)$ \\
\hline$\$ 10000-\$ 24999$ & 59 & 79.10 & (68.54-86.79) & 55 & 79.79 & (71.14-86.35) & 54 & 71.80 & (55.73-83.73) \\
\hline$\$ 25000-\$ 49999$ & 62 & 79.42 & (67.52-87.75) & 55 & 73.00 & $(61.16-82.27)$ & 60 & 78.16 & $(67.16-86.23)$ \\
\hline$\$ 50000-\$ 99999$ & 86 & 77.94 & (68.67-85.06) & 76 & 71.06 & (61.46-79.08) & 79 & 71.29 & (61.95-79.10) \\
\hline$\$ 100000$ or more & 68 & 77.26 & $(61.90-87.67)$ & 66 & 76.47 & (63.46-85.88) & 61 & 72.53 & $(59.63-82.51)$ \\
\hline
\end{tabular}

\begin{tabular}{|c|c|c|c|c|c|c|c|c|c|}
\hline & \multicolumn{3}{|c|}{$\begin{array}{l}\text { It helps people to quit } \\
\text { smoking cigarettes }\end{array}$} & \multicolumn{3}{|c|}{$\begin{array}{l}\text { They can be used in places } \\
\text { where smoking can't }\end{array}$} & \multicolumn{3}{|c|}{$\begin{array}{l}\text { They don't bother } \\
\text { non tobacco users }\end{array}$} \\
\hline & $\mathbf{N}$ & $\%$ & $95^{\circ} \% \mathrm{CI}$ & $\mathrm{N}$ & $\%$ & $95 \% \mathrm{CI}$ & $\mathbf{N}$ & $\%$ & $95^{\circ} \circ \mathrm{CI}$ \\
\hline Overall & 162 & 63.48 & $(57.22-69.32)$ & 242 & 58.81 & (53.36-64.05) & 229 & 56.31 & $(50.65-61.81)$ \\
\hline \multicolumn{10}{|l|}{ Age (years) } \\
\hline $12-14$ & 31 & 64.75 & $(50.33-76.91)$ & 51 & 60.11 & $(48.71-70.51)$ & 45 & 52.41 & $(41.32-63.26)$ \\
\hline $15-17$ & 131 & 63.20 & $(56.76-69.21)$ & 191 & 58.33 & (52.33-64.10) & 184 & 57.39 & $(51.06-63.49)$ \\
\hline \multicolumn{10}{|l|}{ Gender } \\
\hline Male & 96 & 68.70 & (59.54-76.61) & 145 & 60.81 & $(52.65-68.41)$ & 133 & 57.54 & $(50.55-64.24)$ \\
\hline Female & 66 & 57.58 & $(48.27-66.39)$ & 97 & 56.16 & $(49.32-62.77)$ & 95 & 54.68 & (45.90-63.18) \\
\hline \multicolumn{10}{|l|}{ Race/Ethnicity } \\
\hline Non-Hispanic White & 100 & 60.51 & $(52.82-67.72)$ & 155 & 60.00 & (53.15-66.49) & 146 & 56.09 & $(48.29-63.60)$ \\
\hline Non-Hispanic Black & 7 & 83.42 & $(48.10-96.47)$ & 10 & 48.39 & $(22.39-75.28)$ & 11 & 65.32 & $(40.20-84.07)$ \\
\hline Other race & 17 & 67.83 & $(42.71-85.64)$ & 25 & 59.39 & $(42.56-74.27)$ & 21 & 54.25 & (36.62-70.88) \\
\hline Hispanic & 38 & 69.52 & $(55.02-80.96)$ & 52 & 56.36 & (45.67-66.49) & 51 & 55.90 & $(45.63-65.69)$ \\
\hline \multicolumn{10}{|l|}{ Household Income } \\
\hline$<\$ 10000$ & 13 & 77.36 & (47.60-92.78) & 14 & 44.26 & $(27.61-62.30)$ & 15 & 45.71 & $(27.19-65.51)$ \\
\hline$\$ 10000-\$ 24999$ & 39 & 68.42 & $(51.14-81.77)$ & 49 & 68.39 & $(54.87-79.37)$ & 44 & 59.42 & $(45.45-72.01)$ \\
\hline$\$ 25000-\$ 49999$ & 35 & 63.61 & $(49.71-75.55)$ & 43 & 55.76 & $(43.25-67.58)$ & 43 & 56.12 & $(42.81-68.60)$ \\
\hline$\$ 50000-\$ 99999$ & 37 & 60.43 & $(47.10-72.37)$ & 62 & 57.04 & $(46.70-66.80)$ & 61 & 54.85 & $(44.87-64.46)$ \\
\hline$\$ 100000$ or more & 28 & 60.87 & (44.98-74.75) & 48 & 57.24 & (43.17-70.23) & 42 & 54.57 & $(40.90-67.59)$ \\
\hline
\end{tabular}


Table 2. Continued

\begin{tabular}{|c|c|c|c|c|c|c|c|c|c|}
\hline & \multicolumn{3}{|c|}{ They don't smell } & \multicolumn{3}{|c|}{ They are affordable } & \multicolumn{3}{|c|}{$\begin{array}{c}\text { I like socialiving while using } \\
\text { them }\end{array}$} \\
\hline & $\mathbf{N}$ & $\%$ & $95 \%$ CI & $\mathbf{N}$ & $\%$ & $95 \% \mathrm{CI}$ & $\mathbf{N}$ & $\%$ & $95^{\circ} \% \mathrm{CI}$ \\
\hline Overall & 229 & 53.70 & $(48.48-58.83)$ & 208 & 48.07 & $(43.79-52.37)$ & 193 & 45.68 & $(41.50-49.92)$ \\
\hline \multicolumn{10}{|l|}{ Age (years) } \\
\hline $12-14$ & 40 & 42.83 & $(32.72-53.58)^{*}$ & 43 & 46.30 & $(35.65-57.30)$ & 34 & 37.20 & (28.14-47.26) \\
\hline $15-17$ & 189 & 56.46 & $(50.47-62.26)^{*}$ & 165 & 48.64 & $(43.28-54.02)$ & 159 & 47.86 & $(42.56-53.20)$ \\
\hline \multicolumn{10}{|l|}{ Gender } \\
\hline Male & 125 & 51.38 & $(44.84-57.88)$ & 132 & 53.37 & $(46.46-60.17)$ & 112 & 44.44 & $(38.31-50.73)$ \\
\hline Female & 103 & 56.75 & $(49.42-63.79)$ & 75 & 40.96 & $(32.81-49.64)$ & 80 & 47.33 & $(40.29-54.48)$ \\
\hline \multicolumn{10}{|l|}{ Race/Ethnicity } \\
\hline Non-Hispanic White & 142 & 53.02 & $(45.85-60.06)$ & 119 & 45.02 & $(39.89-50.26)$ & 122 & 45.21 & $(40.31-50.21)$ \\
\hline Non-Hispanic Black & 9 & 41.57 & $(21.55-64.82)$ & 8 & 36.85 & $(16.20-63.77)$ & 6 & 30.63 & $(11.72-59.48)$ \\
\hline Other race & 28 & 66.84 & $(49.25-80.71)$ & 28 & 63.18 & (46.34-77.32) & 17 & 44.34 & (26.94-63.25) \\
\hline Hispanic & 50 & 54.48 & $(42.12-66.31)$ & 53 & 57.53 & $(45.70-68.56)$ & 48 & 53.96 & $(42.93-64.63)$ \\
\hline \multicolumn{10}{|l|}{ Household Income } \\
\hline$<\$ 10000$ & 14 & 44.27 & (25.15-65.25) & 14 & 38.44 & $(22.45-57.40)$ & 13 & 38.60 & $(22.29-57.96)$ \\
\hline$\$ 10000-\$ 24999$ & 42 & 54.14 & $(41.75-66.03)$ & 36 & 45.81 & $(33.11-59.08)$ & 35 & 47.68 & $(35.85-59.77)$ \\
\hline$\$ 25000-\$ 49999$ & 42 & 53.85 & $(41.28-65.94)$ & 36 & 44.40 & $(32.73-56.73)$ & 31 & 40.88 & (29.70-53.08) \\
\hline \$50000-\$99999 & 60 & 52.70 & $(44.02-61.22)$ & 57 & 48.78 & $(39.51-58.14)$ & 59 & 51.26 & $(40.70-61.71)$ \\
\hline$\$ 100000$ or more & 51 & 58.30 & $(45.57-70.01)$ & 43 & 50.76 & (38.02-63.39) & 39 & 43.73 & $(31.83-56.40)$ \\
\hline
\end{tabular}

\begin{tabular}{|c|c|c|c|c|c|c|c|c|c|}
\hline & \multicolumn{3}{|c|}{$\begin{array}{c}\text { People in the media or other } \\
\text { public figures use them }\end{array}$} & \multicolumn{3}{|c|}{$\begin{array}{c}\text { People who are important to } \\
\text { me use them }\end{array}$} & \multicolumn{3}{|c|}{$\begin{array}{c}\text { It feels like smoking a regular } \\
\text { cigarette }\end{array}$} \\
\hline & N & $\%$ & $95 \%$ CI & $\mathbf{N}$ & $\%$ & $95^{\circ} \circ \mathrm{CI}$ & $\mathbf{N}$ & $\%$ & $95^{\circ} \% \mathrm{CI}$ \\
\hline Overall & 150 & 36.19 & (30.98-41.74) & 143 & 34.74 & $(29.56-40.31)$ & 107 & 25.15 & $(20.35-30.64)$ \\
\hline \multicolumn{10}{|l|}{ Age (years) } \\
\hline $12-14$ & 39 & 45.10 & $(34.61-56.04)$ & 31 & 34.86 & $(24.60-46.75)$ & 23 & 25.68 & (16.63-37.44) \\
\hline $15-17$ & 111 & 33.93 & $(28.33-40.02)$ & 112 & 34.89 & $(29.05-41.22)$ & 84 & 24.94 & $(20.01-30.63)$ \\
\hline \multicolumn{10}{|l|}{ Gender } \\
\hline Male & 86 & 34.78 & $(28.35-41.83)$ & 67 & 28.24 & $(22.63-34.63)^{* *}$ & 52 & 20.49 & $(15.68-26.32)^{*}$ \\
\hline Female & 64 & 38.06 & $(29.98-46.86)$ & 75 & 43.28 & $(35.62-51.27)^{* *}$ & 55 & 31.30 & $(23.46-40.36)^{*}$ \\
\hline \multicolumn{10}{|l|}{ Race/Ethnicity } \\
\hline Non-Hispanic White & 88 & 33.91 & $(28.01-40.36)$ & 98 & 36.12 & $(30.25-42.44)$ & 67 & 24.70 & $(18.71-31.86)$ \\
\hline Non-Hispanic Black & 7 & 26.77 & $(9.28-56.62)$ & 6 & 29.58 & $(13.18-53.75)$ & 8 & 37.13 & (18.64-60.35) \\
\hline Other race & 18 & 47.77 & (30.13-65.99) & 17 & 44.34 & $(28.13-61.85)$ & 13 & 30.46 & (17.98-46.68) \\
\hline Hispanic & 37 & 42.63 & $(32.96-52.91)$ & 22 & 26.62 & $(18.62-36.51)$ & 19 & 20.07 & (13.59-28.61) \\
\hline \multicolumn{10}{|l|}{ Household Income } \\
\hline$<\$ 10000$ & 13 & 39.31 & $(21.41-60.63)$ & 8 & 26.72 & $(12.88-47.36)$ & 9 & 27.16 & $(12.64-49.01)^{*}$ \\
\hline$\$ 10000-\$ 24999$ & 34 & 47.66 & (35.99-59.59) & 22 & 26.33 & $(17.31-37.91)$ & 24 & 32.86 & $(22.84-44.73)^{*}$ \\
\hline$\$ 25000-\$ 49999$ & 28 & 38.73 & $(27.35-51.48)$ & 25 & 34.49 & $(23.67-47.20)$ & 22 & 27.50 & $(18.70-38.48)^{*}$ \\
\hline$\$ 50000-\$ 99999$ & 31 & 27.92 & $(19.44-38.34)$ & 40 & 37.60 & $(29.24-46.77)$ & 28 & 28.15 & $(19.64-38.57)^{*}$ \\
\hline$\$ 100000$ or more & 29 & 32.44 & $(22.35-44.49)$ & 33 & 37.38 & $(26.71-49.45)$ & 13 & 13.80 & $(8.58-21.46)^{*}$ \\
\hline
\end{tabular}


Table 2. Continued

\begin{tabular}{|c|c|c|c|}
\hline & \multicolumn{3}{|c|}{ Advertising appeals to me } \\
\hline & $\mathbf{N}$ & $\%$ & $95^{\circ} \% \mathrm{CI}$ \\
\hline Overall & 60 & 14.18 & $(10.63-18.66)$ \\
\hline \multicolumn{4}{|l|}{ Age (years) } \\
\hline $12-14$ & 20 & 21.50 & $(13.13-33.16)$ \\
\hline $15-17$ & 40 & 12.37 & $(8.83-17.06)$ \\
\hline \multicolumn{4}{|l|}{ Gender } \\
\hline Male & 38 & 14.45 & $(10.01-20.40)$ \\
\hline Female & 22 & 13.90 & $(9.02-20.80)$ \\
\hline \multicolumn{4}{|l|}{ Race/Ethnicity } \\
\hline Non-Hispanic White & 35 & 13.02 & $(8.91-18.64)$ \\
\hline Non-Hispanic Black & 4 & 17.05 & $(5.21-43.48)$ \\
\hline Other race & 5 & 14.35 & $(5.44-32.76)$ \\
\hline Hispanic & 16 & 17.87 & (10.69-28.35) \\
\hline \multicolumn{4}{|l|}{ Household Income } \\
\hline$<\$ 10000$ & 6 & 16.27 & $(7.17-32.85)^{* *}$ \\
\hline$\$ 10000-\$ 24999$ & 18 & 26.42 & $(16.65-39.22)^{* *}$ \\
\hline$\$ 25000-\$ 49999$ & 12 & 15.03 & $(7.80-27.01)^{* *}$ \\
\hline$\$ 50000-\$ 99999$ & 13 & 11.45 & $(6.65-19.03)^{* *}$ \\
\hline$\$ 100000$ or more & 4 & 4.34 & $(1.56-11.51)^{* *}$ \\
\hline
\end{tabular}

other non-Hispanic ethnicities (89.3\%). Among older adolescents (15-17 years old), $56.5 \%$ reported using ENDS because the products do not smell bad, compared to $42.8 \%$ of younger adolescents (12-14 years old). Among those with household incomes of less than $\$ 10000$ a year, $16.3 \%$ reported using ENDS because the advertising appealed to them, compared to $26.4 \%$ of those with household incomes of $\$ 10000$ $\$ 24999,15.0 \%$ of those with household incomes of \$25000-\$49999, 11.5\% of those with household incomes of $\$ 50000-\$ 99999$ and $4.34 \%$ of those with household incomes of $\$ 100000$ or more.

Logistic regression analyses (Table 3 ) indicated that variations in use behavior were associated with several motivations for ENDS use. These analyses indicate that compared to non-Hispanic Whites, those of nonHispanic ethnicities other than non-Hispanic White or Black had a higher likelihood of reporting using ENDS because they are affordable $(\mathrm{AOR}=2.684,95 \%$ CI: 1.044-6.899), and females had a higher likelihood of reporting using ENDS because people important to them use ENDS (AOR=1.895, 95\% CI: 1.128$3.184)$ and a lower likelihood of reporting using ENDS because they are affordable (AOR $=0.538,95 \%$

Table 3. Adjusted odds ratios for association between sociodemographics and tobacco variables with motivations for e- cigarette use, United States, 2014-2016 ( $\mathrm{N}=415)$

\begin{tabular}{|c|c|c|c|c|c|c|c|c|}
\hline & \multicolumn{2}{|c|}{$\begin{array}{l}\text { It comes in flavors } \\
\text { I like }\end{array}$} & \multicolumn{2}{|c|}{$\begin{array}{l}\text { They might be less } \\
\text { harmful to me } \\
\text { than cigarettes }\end{array}$} & \multicolumn{2}{|c|}{$\begin{array}{l}\text { They might be less } \\
\text { harmitul to people } \\
\text { around me }\end{array}$} & \multicolumn{2}{|c|}{$\begin{array}{l}\text { It helps people } \\
\text { to quit smoking } \\
\text { cigarettes }\end{array}$} \\
\hline & AOR & $95 \% \mathrm{CI}$ & AOR & $95^{\circ} \circ \mathrm{CI}$ & IOR & $95^{\circ} \% \mathrm{CI}$ & AOR & $95^{\circ} \% \mathrm{CI}$ \\
\hline 15-17 years old (ref: $12-14$ years old) & 0.590 & $(0.291-1.198)$ & 0.608 & $(0.295-1.25)$ & 0.501 & $(0.244-1.030)$ & 0.668 & $(0.308-1.449)$ \\
\hline Female (ref: Male) & 1.751 & $(0.928-3.305)$ & 0.992 & $(0.577-1.707)$ & 1.415 & $(0.781-2.564)$ & 0.636 & $(0.337-1.202)$ \\
\hline \multicolumn{9}{|l|}{ Race/ethnicity (ref: Non-Hispanic White) } \\
\hline Non-Hispanic Black & 0.512 & $(0.117-2.236)$ & 4.818 & $(0.623-37.275)$ & 2.594 & $(0.688-9.778)$ & 4.221 & $(0.262-68.03)$ \\
\hline Other race & 1.875 & $(0.593-5.929)$ & 1.309 & $(0.489-3.503)$ & 2.093 & $(0.732-5.986)$ & 1.702 & $(0.421-6.882)$ \\
\hline Hispanic & 1.000 & $(0.510-1.958)$ & 0.648 & $(0.328-1.279)$ & 1.287 & $(0.572-2.894)$ & 1.174 & $(0.506-2.723)$ \\
\hline \multicolumn{9}{|l|}{ Household income (ref: $<\$ 10000 /$ year) } \\
\hline$\$ 10000-\$ 24999$ & 1.385 & $(0.481-3.984)$ & 1.757 & $(0.612-5.044)$ & 0.838 & $(0.252-2.788)$ & 0.625 & $(0.087-4.497)$ \\
\hline$\$ 25000-\$ 49999$ & 1.516 & $(0.533-4.316)$ & 1.116 & $(0.377-3.301)$ & 1.188 & $(0.349-4.040)$ & 0.435 & $(0.064-2.93)$ \\
\hline$\$ 50000-\$ 99999$ & 1.222 & $(0.433-3.449)$ & 1.025 & $(0.365-2.878)$ & 0.773 & $(0.214-2.788)$ & 0.402 & $(0.054-2.999)$ \\
\hline$\$ 100000$ or more & 1.255 & $(0.388-4.061)$ & 1.464 & $(0.441-4.857)$ & 0.972 & $(0.255-3.704)$ & 0.427 & $(0.056-3.277)$ \\
\hline $\begin{array}{l}\text { Uses a flavored e-cigarette (ref: does } \\
\text { not use flavored e-cigarette) }\end{array}$ & 2.300 & $(1.153-4.585)^{*}$ & 1.220 & $(0.642-2.316)$ & 1.976 & $(1.082-3.605)^{*}$ & 1.978 & $(0.89-4.395)$ \\
\hline $\begin{array}{l}\text { Combustible tobacco use during Wave } 2 \\
\text { (ref: not during Wave 2) }\end{array}$ & 1.416 & $(0.746-2.687)$ & 1.525 & $(0.855-2.717)$ & 1.337 & $(0.735-2.434)$ & 1.275 & $(0.47-3.462)$ \\
\hline Days used e-cigarette in past 30 days & 1.035 & $(0.994-1.078)$ & 1.025 & $(0.993-1.058)$ & 1.058 & $(1.022-1.095)^{* *}$ & 1.011 & $(0.976-1.047)$ \\
\hline
\end{tabular}


Table 3. Continued

\begin{tabular}{|c|c|c|c|c|c|c|c|c|}
\hline & \multicolumn{2}{|c|}{$\begin{array}{l}\text { They can be used } \\
\text { in places where } \\
\text { smoking can't }\end{array}$} & \multicolumn{2}{|c|}{$\begin{array}{l}\text { They don't bother } \\
\text { non-tobacco users }\end{array}$} & \multicolumn{2}{|c|}{ They don't smell } & \multicolumn{2}{|c|}{ They are affordable } \\
\hline & IOR & $95^{\circ} \% \mathrm{CI}$ & AOR & $95^{\circ} \mathrm{OCI}$ & AOR & $95^{\circ} \mathrm{CI}$ & IOR & $95^{\circ} \mathrm{CI}$ \\
\hline 15-17 years old (ref: $12-14$ years old) & 0.767 & $(0.398-1.478)$ & 1.167 & $(0.649-2.098)$ & 1.595 & $(0.829-3.069)$ & 0.856 & $(0.463-1.584)$ \\
\hline Female (ref: Male) & 0.867 & $(0.524-1.435)$ & 0.916 & $(0.548-1.563)$ & 1.216 & $(0.752-1.965)$ & 0.538 & $(0.296-0.976)^{*}$ \\
\hline \multicolumn{9}{|l|}{ Race/ethnicity (ref: Non-Hispanic White) } \\
\hline Non-Hispanic Black & 1.111 & $(0.271-4.550)$ & 1.998 & $(0.610-6.552)$ & 0.762 & $(0.221-2.630)$ & 1.039 & $(0.234-4.610)$ \\
\hline Other race & 1.005 & $(0.386-2.619)$ & 1.022 & $(0.440-2.374)$ & 1.715 & $(0.699-4.208)$ & 2.684 & $(1.044-6.899)^{*}$ \\
\hline Hispanic & 0.835 & $(0.442-1.579)$ & 0.913 & $(0.447-1.865)$ & 1.632 & $(0.780-3.415)$ & 1.934 & $(0.995-3.759)$ \\
\hline \multicolumn{9}{|l|}{ Household income (ref: $<\$ 10000 /$ year) } \\
\hline$\$ 10000-\$ 24999$ & 2.221 & $(0.772-6.387)$ & 1.837 & $(0.615-5.487)$ & 1.457 & $(0.509-4.347)$ & 1.119 & $(0.369-3.395)$ \\
\hline$\$ 25000-\$ 49999$ & 1.192 & $(0.408-3.481)$ & 1.420 & $(0.451-4.476)$ & 1.635 & $(0.543-4.922)$ & 1.186 & $(0.382-3.675)$ \\
\hline$\$ 50000-\$ 99999$ & 1.345 & $(0.515-3.512)$ & 1.280 & $(0.500-3.278)$ & 1.361 & $(0.450-4.118)$ & 1.412 & $(0.476-4.191)$ \\
\hline$\$ 100000$ or more & 1.466 & $(0.512-4.198)$ & 1.325 & $(0.522-3.364)$ & 1.869 & $(0.572-6.110)$ & 1.670 & $(0.527-5.299)$ \\
\hline $\begin{array}{l}\text { Uses a flavored e-cigarette (ref: does } \\
\text { not use flavored e-cigarette) }\end{array}$ & 1.663 & $(0.915-3.019)$ & 1.252 & $(0.695-2.258)$ & 1.586 & $(0.764-3.291)$ & 1.827 & $(0.982-3.400)$ \\
\hline $\begin{array}{l}\text { Combustible tobacco use during Wave } 2 \\
\text { (ref: not during Wave 2) }\end{array}$ & 2.340 & $(1.431-3.828)^{* *}$ & 1.118 & $(0.714-1.751)$ & 1.740 & $(1.117-2.711)^{*}$ & 1.483 & $(0.892-2.466)$ \\
\hline Days used e-cigarette in past 30 days & 1.061 & $(1.031-1.093)^{* * *}$ & 1.037 & $(1.004-1.070)^{*}$ & 1.012 & $(0.983-1.042)$ & 1.023 & $(0.995-1.051)$ \\
\hline
\end{tabular}

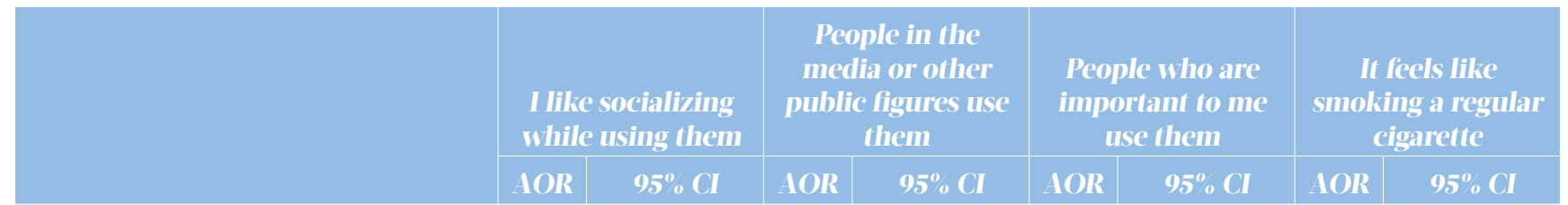

15-17 years old (ref: 12-14 years old) Female (ref: Male)

Race/ethnicity (ref: Non-Hispanic White)

Non-Hispanic Black

Other race

Hispanic

Household income (ref: $<\$ 10000 /$ year)

\$10000-\$24999

\$25000-\$49999

\$50000-\$99999

$\$ 100000$ or more

Uses a flavored e-cigarette (ref: does not use flavored e-cigarette)

Combustible tobacco use during Wave 2 (ref: not during Wave 2)

Days used e-cigarette in past 30 days

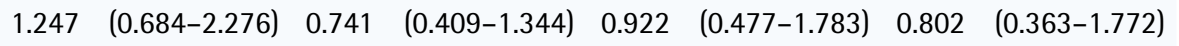

$1.212(0.743-1.976) 1.193(0.668-2.132) 1.895 \quad(1.128-3.184)^{*} 1.761 \quad(1.060-2.927)^{*}$

$\begin{array}{llllllll}0.807 & (0.188-3.464) & 0.812 & (0.212-3.101) & 0.997 & (0.307-3.236) & 2.740 & (1.000-7.513)^{*}\end{array}$

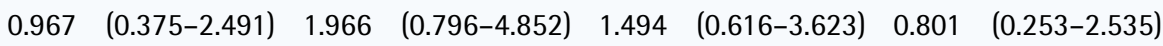

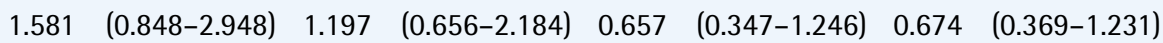

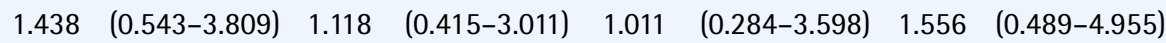

$\begin{array}{lllllllll}1.042 & (0.365-2.978) & 0.904 & (0.304-2.689) & 1.513 & (0.438-5.224) & 1.142 & (0.366-3.560)\end{array}$

$\begin{array}{llllllll}1.426 & (0.623-3.261) & 0.521 & (0.181-1.502) & 1.444 & (0.476-4.382) & 1.310 & (0.458-3.748)\end{array}$

$\begin{array}{lllllllll}1.138 & (0.391-3.312) & 0.728 & (0.244-2.166) & 1.439 & (0.463-4.476) & 0.497 & (0.168-1.467)\end{array}$

$2.221 \quad(1.280-3.853)^{* *} 1.208 \quad(0.64-2.281) \quad 3.005 \quad(1.532-5.892)^{* *} 0.947 \quad(0.393-2.285)$

$1.157(0.701-1.910) \quad 1.177 \quad(0.705-1.963) \quad 0.874 \quad(0.524-1.455) \quad 3.810 \quad(2.079-6.979)^{* * *}$ $1.033 \quad(1.003-1.064)^{*} \quad 1.006 \quad(0.978-1.034) \quad 1.012 \quad(0.985-1.040) \quad 1.008 \quad(0.975-1.042)$ 
Table 3. continued

\begin{tabular}{|c|c|c|}
\hline & \multicolumn{2}{|c|}{$\begin{array}{l}\text { The advertising } \\
\text { appeals to me }\end{array}$} \\
\hline & AOR & $95^{\circ} \mathrm{COI}$ \\
\hline 15-17 years old (ref: $12-14$ years old) & 0.488 & $(0.194-1.225)$ \\
\hline Female (ref: Male) & 0.807 & $(0.393-1.656)$ \\
\hline \multicolumn{3}{|l|}{ Race/ethnicity (ref: Non-Hispanic White) } \\
\hline Non-Hispanic Black & 2.542 & $(0.523-12.348)$ \\
\hline Other race & 0.884 & $(0.154-5.091)$ \\
\hline Hispanic & 1.493 & $(0.662-3.368)$ \\
\hline \multicolumn{3}{|l|}{ Household income (ref: $<\$ 10000 /$ year) } \\
\hline$\$ 10000-\$ 24999$ & 1.763 & $(0.380-8.168)$ \\
\hline$\$ 25000-\$ 49999$ & 0.918 & $(0.210-4.006)$ \\
\hline$\$ 50000-\$ 99999$ & 0.817 & $(0.223-3.000)$ \\
\hline$\$ 100000$ or more & 0.289 & $(0.068-1.220)$ \\
\hline $\begin{array}{l}\text { Uses a flavored e-cigarette (ref: does } \\
\text { not use flavored e-cigarette) }\end{array}$ & 0.900 & $(0.423-1.913)$ \\
\hline $\begin{array}{l}\text { Combustible tobacco use during Wave } 2 \\
\text { (ref: not during Wave 2) }\end{array}$ & 2.589 & $(1.301-5.151)^{* *}$ \\
\hline Days used e-cigarette in past 30 days & 1.003 & $(0.960-1.048)$ \\
\hline
\end{tabular}

CI: 0.296-0.976). Combustible tobacco users had a higher likelihood of reporting using ENDS, because they compared favorably to cigarettes in that they do not smell, can be used in places cigarettes are not allowed, and feel like smoking a cigarette. Flavored ENDS users had a higher likelihood of reporting using ENDS due to the appealing flavors, reduced harm perceptions, and use in socialization or because people important to them use ENDS. Adolescents who use ENDS more intensely had a higher likelihood of reporting socialization, reduced harm to others, and not bothering other people as reasons for use.

\section{DISCUSSION}

Appealing flavors was the most commonly reported motivation for using ENDS, but was mentioned more often among females. It is possible that flavors may be especially attractive to adolescent girls, as similar preferences for flavored products are observed in other products such as alcohol ${ }^{16}$. Perceptions of reduced harm to self and to others were the next most commonly reported reasons for ENDS use across all youth. This reason did not differ across sociodemographic groups, indicating that perceptions of reduced harm are important for most adolescent
ENDS users, regardless of sociodemographic characteristics. Advertising was not commonly reported as a reason for ENDS use.

Logistic regression analyses revealed that similarity or exchangeability with cigarettes is an important reason for ENDS use among combustible tobacco users. Further research should explore whether ENDS are being used as an alternative to combustible tobacco among this group. More intense ENDS use was associated with reasons involving other people, indicating that social benefit may encourage more intense use.

These findings offer several insights that could be helpful for policy and practice. First, given flavor's importance in motivating product use, limiting appealing flavors may be an important approach to reduce use among youth, particularly female youth. Second, measures to increase the price of ENDS may have more of an effect on those of other non-Hispanic ethnicities compared to non-Hispanic Whites, as this group had a higher likelihood of reporting affordability as a reason for ENDS use. Conversely, price-increasing measures may have less of an effect on females compared to males, as females were less likely to report affordability as a motivation for ENDS use. Additionally, given the high number of youth overall who report using ENDS because they believe they are less harmful to them than cigarettes, education campaigns should communicate the potential harms of ENDS. Finally, given the prevalence of youth reporting using ENDS because people who are close to them use them, campaigns or interventions that leverage the role of important people in youths' lives may be a useful strategy.

Several limitations must be considered when interpreting our findings. First, individuals may be more likely to select those motivations that come quickly to mind. With no opportunity to report motivations outside those included in the PATH study questionnaire, other important motivations may not be reflected in the study and this analysis. Second, only youth who had used ENDS in the past 30 days were asked questions about motivations for use. Therefore, we cannot draw conclusions about motivations for use among individuals outside this group. Moreover, this sample size was relatively small. While weighting procedures were employed, future work should be done with larger samples. Finally, data on specific racial/ethnic groups other than non-Hispanic White, non-Hispanic Black and Hispanic were not available in the data file, so we are 
unable to draw conclusions about motivations for ENDS use among these specific populations.

\section{CONCLUSIONS}

A long history of health disparities in tobacco product use $^{17}$ underscores the need for tobacco control policies and interventions that effectively reduce ENDS use among vulnerable subpopulations of adolescents. Understanding the motivations for use among sociodemographic subgroups is an initial step towards informing the development of policies and interventions with equally distributed benefits.

\section{REFERENCES}

1. Wang TW, Gentzke A, Sharapova S, Cullen KA, Ambrose B, Jamal A. Tobacco Use Among Middle and High School Students - United States, 2011-2017. MMWR Morb Mortal Wkly Rep. 2018;67(22):629-633. doi:10.15585/mmwr.mm6722a3

2. Kong G, Kuguru KE, Krishnan-Sarin S. Gender Differences in US Adolescent E-Cigarette Use. Current Addiction Reports. 2017;4(4)422-430. doi:10.15585/mmwr.mm6722a3

3. Simon P, Camenga D, Morean M, et al. Socioeconomic status and adolescent e-cigarette use: The mediating role of e-cigarette advertisement exposure. Preventive Medicine. 2018;112:193-198. doi:10.1016/j.ypmed.2018.04.019

4. Anand V, McGinty KL, O'Brien K, Guenthner G, Hahn E, Martin CA. E-cigarette use and beliefs among urban public high school students in North Carolina. Journal of Adolescent Health. 2015;57(1):46-51. doi:10.1016/j.jadohealth.2015.03.018

5. McNeill A, Brose LS, Calder R, Hitchman SC, Hajek P, McRobbie H. E-cigarettes: an evidence update. Public Health England. https://assets.publishing.service.gov.uk/ government/uploads/system/uploads/attachment_data/ file/457102/Ecigarettes_an_evidence_update_A_report_ commissioned_by_Public_Health_England_FINAL.pdf. Published August 19, 2015. Updated August 28, 2015. Accessed June 16, 2018.

6. Cheng T. Chemical evaluation of electronic cigarettes. Tobacco Control. 2014;23(2):ii11-ii17. doi:10.1136/tobaccocontrol-2013-051482

7. Dwyer JB, McQuown SC, Leslie FM. The Dynamic Effects of Nicotine on the Developing Brain. Pharmacology \& Therapeutics. 2009;122(2):125-139. doi:10.1016/j.pharmthera.2009.02.003

8. Arrazola R, Dube SR, King BA. Tobacco product use middle and high school students- United States, 2011-2012. MMWR Morb Mortal Wkly Rep. 2013;62(45):893-897.

9. Cooper M, Harrell MB, Perry CL. Comparing young adults to older adults in e-cigarette perceptions and motivations for use: implications for health communication.
Health Education Research. 2016;31(4):429-438. doi:10.1093/her/cyw030

10. Villanti AC, Johnson AL, Ambrose BK, et al. Flavored tobacco product use in youth and adults: Findings from the first wave of the PATH study (2013-2014). American Journal of Preventive Medicine. 2017;53(2):139-151. doi:10.1016/j.amepre.2017.01.026

11. Kong G, Morean ME, Cavallo DA, Camenga DR, KrishnanSarin S. Reasons for Electronic Cigarette Experimentation and Discontinuation Among Adolescents and Young Adults. Nicotine \& Tobacco Research. 2015;17(7):847854. doi:10.1093/ntr/ntu257

12. Pulvers K, Scheuermann TS, Emami AS, et al. Reasons for Smoking Among Tri-Ethnic Daily and Nondaily Smokers. Nicotine \& Tobacco Research. 2014;16(12):1567-1576. doi: $10.1093 / \mathrm{ntr} / \mathrm{ntu} 108$

13. Patel D, Davis KC, Cox S, et al. Reasons for current E-cigarette use among US adults. Preventive Medicine. 2016;93:14-20. doi:10.1016/j.ypmed.2016.09.011

14. Pepper JK, Ribisl KM, Emery SL, Brewer NT. Reasons for starting and stopping electronic cigarette use. International Journal of Environmental Research and Public Health. 2014;11(10):10345-10361. doi:10.3390/ijerph111010345

15. Hyland A, Ambrose BK, Conway KP, Borek N, Lambert E, Carusi C, et al. Design and methods of the Population Assessment of Tobacco and Health (PATH) Study. Tobacco Control. 2016;26(4):371-378. doi:10.1136/tobaccocontrol-2016-052934

16. Windle M. Drinking Over the Lifespan: Focus on Early Adolescents and Youth. Alcohol Research: Current Reviews. 2016;38(1):95-101.

17. U.S. National Cancer Institute. A Socioecological Approach to Addressing Tobacco Related Health Disparities. National Cancer Institute Tobacco Control Monograph 22. NIH Publication No. 17-CA-8035A. Bethesda, MD: U.S. Department of Health and Human Services, National Institutes of Health, National Cancer Institute; 2017.

\section{CONFLICTS OF INTEREST}

The authors declare that they have no competing interests, financial or otherwise, related to the current work. M. B. Moran reports grants from National Institute on Drug Abuse/US Food and Drug Administration Center for Tobacco Products, during the conduct of the study. The rest of the authors have also completed and submitted an ICMJE form for disclosure of potential conflicts of interest.

\section{FUNDING}

Dr. Moran's effort is supported by NIDA and FDA Center for Tobacco Products (CTP) (K01DA037903, PI: Moran). The content is solely the responsibility of the authors and does not necessarily represent the official views of the NIH or the Food and Drug Administration.

\section{PROVENANCE AND PEER REVIEW}

Not commissioned; externally peer reviewed. 